\title{
Effect of voluntary contraction of the masseter and other muscles upon the masseteric reflex in man
}

\author{
A. G. HANNAM \\ From the Department of Oral Biology, Faculty of Dentistry, \\ University of British Columbia, Vancouver 8, Canada
}

SUMMARY Experiments were carried out on seven adult subjects in order to establish the relationship between the magnitude of the masseteric reflex and the amount of voluntary activity present in $\frac{\bar{s}^{2}}{\sigma}$ the muscle at the time the reflex was evoked. At the same time, an effort was made to determine $\stackrel{\mathbb{D}}{\circ}$ whether the magnitude of the reflex could be enhanced by the simultaneous voluntary contraction of muscles other than that being tested (the Jendrassik manoeuvre). The reflex was evoked by applying $\vec{O}$ controlled downward thrusts to the mandible so as to produce a constant displacement in each case, $\vec{\overrightarrow{ }}$ and the response of the masseter muscle was recorded by means of small bipolar surface electrodes $\mathscr{\sigma}^{-}$ attached to the skin over the muscle. These responses were averaged by a computer in the presence $\overline{5}$ of various static loads supported by the mandible. It was found that in all subjects the amplitude of the masseteric reflex appeared to increase as the weight supported by the mandible increased, and that in the majority of subjects it was possible to demonstrate Jendrassik facilitation by simultaneow:-

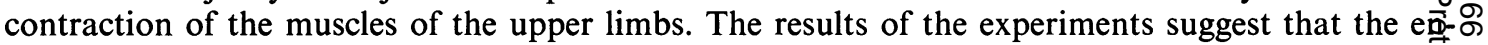
hancement of the masseteric reflex by voluntary contraction of the jaw-closing muscles may be dife을 to autogenetic factors, synergistic factors, or both, and that at least two processes contribute to tamplitude of the masseteric reflex evoked by tooth contact during mastication-namely, the stimul tion of muscle spindles by the impact of opposing teeth and facilitation caused by voluntary activity in the jaw-closing muscles before tooth contact.

The masseteric reflex, otherwise known as the jaw jerk, is a monosynaptic reflex that is readily evoked in man by the rapid downward displacement of the mandible-for example, by a sharp tap applied to the chin. As its name implies, it includes the masseter, the temporal, and probably the medial pterygoid muscles, and results in a rapid rebound displacement of the mandible towards the maxilla. The basic stretch reflex or tendon jerk, of which the masseteric reflex is an example, has in the past received a considerable amount of attention from both neurophysiologists and clinicians. However, there seems to be little information available that relates, in man, the magnitude of the stretch reflex to the amount of voluntary activity already present in the muscle being tested. Knowledge of such a relationship is of some general relevance in that subjects with an anxiety represented by generalized muscle tension often exhibit hyperactive tendon jerk reflexes, yet incomplete relaxation of specific muscles under test frequently results in reflex depression (Ott and
Gassel, 1969). Moreover, an understanding of ${ }^{\circ}$ its particular influence upon the masseteric reflex would help to clarify some of the events associated with the neural control of mastica-o tion. For example, several investigators have $\frac{\mathscr{Q}}{\mathbb{D}}$ suggested that during mastication the pause $\stackrel{\varrho}{\rightarrow}$ which appears in the electrical activity of the음 jaw closing muscles after tooth contact is due to reflex inhibition by the mechanoreceptors of the tooth-supporting structures (Schaerer, Stallard, and Zander, 1967; Brenman, Black, and Coslet, 1968; Ahlgren, 1969; Beaudreau, Daugherty, and Masland, 1969; Griffin and Munro, 1969) 3 . On the other hand, it has been proposed that this effect is mainly due to the excitation of 2 muscle spindles in the temporalis and massetero muscles brought about by the vibration associa- $\rightarrow$ ted with tooth contact or a slight rebound of the을. mandible after contact; this results in a mono-N synaptic reflex followed by the silent period that characteristically follows the superimposition of a stretch reflex on an already contracting muscle ${ }_{\sigma}^{\omega}$ (Hannam, Matthews, and Yemm, 1969, 1970 
Matthews and Yemm, 1970). Hoffman (1922), Hagbarth (1962), and Ott and Gassel (1969) found that a light to moderate amount of voluntary activity in a given muscle facilitates the monosynaptic reflex in the muscle tested, and it was further proposed by Ott and Gassel that, for the ankle jerk, greater degrees of voluntary activity produced inhibition rather than facilitation of the reflex. The present study was undertaken in order to establish the relationship between the magnitude of the masseteric reflex and the amount of voluntary activity present in the muscle at the time the reflex was evoked. At the same time, efforts were made to determine whether the magnitude of the reflex could be enhanced by the simultaneous voluntary contraction of muscles other than that being tested (the Jendrassik manoeuvre).

\section{METHODS}

The present experiments were carried out on seven adult subjects. In each case, the electrical activity in the left masseter muscle was recorded before, during, and after the monosynaptic stretch reflex was elicited in the muscle by applying a controlled downward thrust to the incisal edges of the lower incisor teeth. During this time, the muscle was being voluntarily contracted against known variable loads hung from the incisor teeth so as to provide different degrees of background activity. The apparatus used to evoke the masseteric reflex is illustrated diagrammatically in Fig. 1. It was designed so that the stimulus resulted in a constant displacement of the mandible despite variations in the supported load. The subjects placed their upper and lower incisor teeth in the notches cut in the steel bars ( $\frac{1}{4}$ in. diameter). As can be seen from

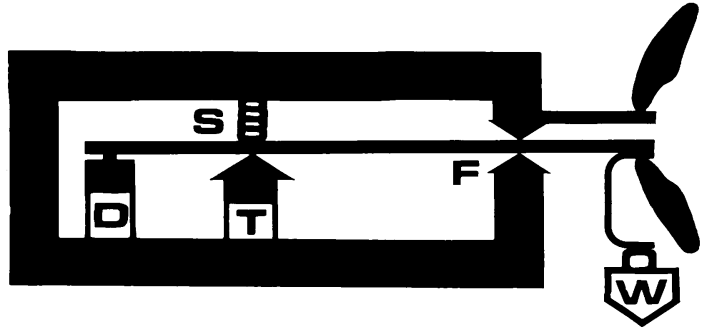

FIG. 1. Diagrammatic representation of the apparatus used to deliver and monitor a regular displacement stimulus to the mandible while at the same time permitting alterations in the load supported by the jaw elevator muscles. The manner in which the displacement transducer $D$, the force generator $F$, the spring $S$, the fulcrum $F$, and the weight $W$ interrelated when the teeth were placed against the bars at the right of the Figure, is described in the text. the diagram, the upper bar was fixed rigidly to the framework which was in turn clamped to a heavy metal table. The lower bar (6 in. long) was allowed to pivot about a fulcrum $(\mathrm{F})$ with the aid of a ballbearing axle located 1 in. from the notched end and attached to the main framework. Controlled movement of the lower bar was accomplished by the opposing action of a thrust $(\mathrm{T})$ delivered by a triggered electromechanical force transducer (Ling vibration generator $\mathrm{V} 47,30 \Omega$ ) and a return spring (S). Displacement of the bar was monitored by a mechanoelectrical transducer (D). This frictionless measuring device consisted of two coils of insulated copper wire, the inner forming a cylinder $\frac{1}{8}$ in. in diameter and 1 in. long and the outer being 1 in. in diameter and 3 in. long, designed so that a $25 \mathrm{kHz}$ current passed through the outer coil would induce in the inner coil a similar current according to the relative positions of the coils. This current could then be demodulated and filtered to produce a DC analogue signal. Calibration showed that the amplitude of the DC signal was linearly related to the displacement of the bar within the limits of movement expected in the experiment, provided that the coils were arranged so that the inner coil moved through the end of the outer. Apart from acting as a monitor of displacement, the signal was also used to trigger a $10 \mathrm{msec}$ current pulse that energized the vibration generator. Thus, during an experimental run, the system worked as follows: each subject was asked to hang a known weight (W), by means of a C-shaped hook, from the incisal edges of his lower anterior teeth. He then placed his upper and lower teeth (the latter covered in part by the hook) in the notches provided and slowly closed his jaws. The displacement sensor output was arranged to trigger the stimulus just before the bar (at this time being depressed against the light spring (S) on the other side of the fulcrum $F$ ) touched the moving element of the vibration generator. The $10 \mathrm{msec}$ pulse from the generator then caused the bar to move briskly in the opposite direction, thus depressing the mandible. The actual pulse to the teeth was light, the most dominant sensation being that caused by the supported weight. When the stimulus was triggered the jaws were separated by $1.5 \mathrm{~cm}$ and by opening his jaws slightly (still maintaining contact with the bars) and then closing them again the subject could reproduce the stimulus at will, thus making the procedure suitable for averaging techniques. Changes in the weight being supported caused slight changes in the amount of displacement of the mandible if the same force were delivered by the vibration generator in each case. However, during the experiments the amplitude of the force was always altered according to the added weight in order to maintain a constant displacement of the mandible, usually about $0.3 \mathrm{~mm}$.

In each subject the response of the left masseter muscle was recorded by means of small surface 
electrodes $2 \mathrm{~mm}$ in diameter attached to the skin over the muscle. The electrodes were placed $1 \mathrm{~cm}$ apart in the longitudinal axis of the muscle fibres, midway between the zygomatic arch and the lower border of the mandible. The signals were amplified by an AC-coupled differential amplifier (Tektronix 3A9) at a bandwidth of $1 \mathrm{~Hz}$ to $1 \mathrm{kHz}$. These responses and the signals representing displacement of the mandible were monitored on a storage oscilloscope (Tektronix 564B) and then led to a signal analyser system (Hewlett-Packard 5481A) that was used to average eight consecutive responses at each test. Tests were carried out in the absence of any added weight other than the hook and the force needed to overcome the return spring (combined resistive force $200 \mathrm{~g}$ ), and with added weights of 1 , 2 , and $3 \mathrm{~kg}$. Weights were added sequentially from 0 to $3 \mathrm{~kg}$ during testing and then removed sequentially from 3 to $0 \mathrm{~kg}$ so that the effect of each of the four variables upon the response of the muscle was evaluated twice. In any one subject, the mean of the two results was then used as a measure of the amplitude of the masseteric reflex for that weight condition, and in this manner data were obtained for all conditions.

In that part of the experiment where an attempt was made to determine whether the masseteric reflex could be influenced by the voluntary contraction of more distant muscles, each subject was first asked to perform the manoeuvre as outlined above in the absence of any supported weights and while sitting comfortably in an upright position. During this time eight responses were averaged. Without changing the experimental arrangement, the subject was then asked to repeat the same procedure while interlocking the fingers of both hands, either in front of his chest or behind his back, and to attempt forcefully to pull them apart. Care was taken to ensure that the displacement of the mandible after the triggered stimulus was the same in both cases, and once again the amplitude of the mean of eight responses was measured.

\section{RESULTS}

The constancy with which both stimuli and responses could be reproduced is shown in Fig. 2 where eight consecutive records have been superimposed and photographed from a storage oscilloscope; in addition, this figure provides, for comparison, a record of the computed means obtained from the signal analyser.

In all subjects the amplitude of the masseteric reflex appeared to increase as the weight supported by the mandible was increased. An example of this effect is shown in Fig. 3 where a set of mean responses at the four different
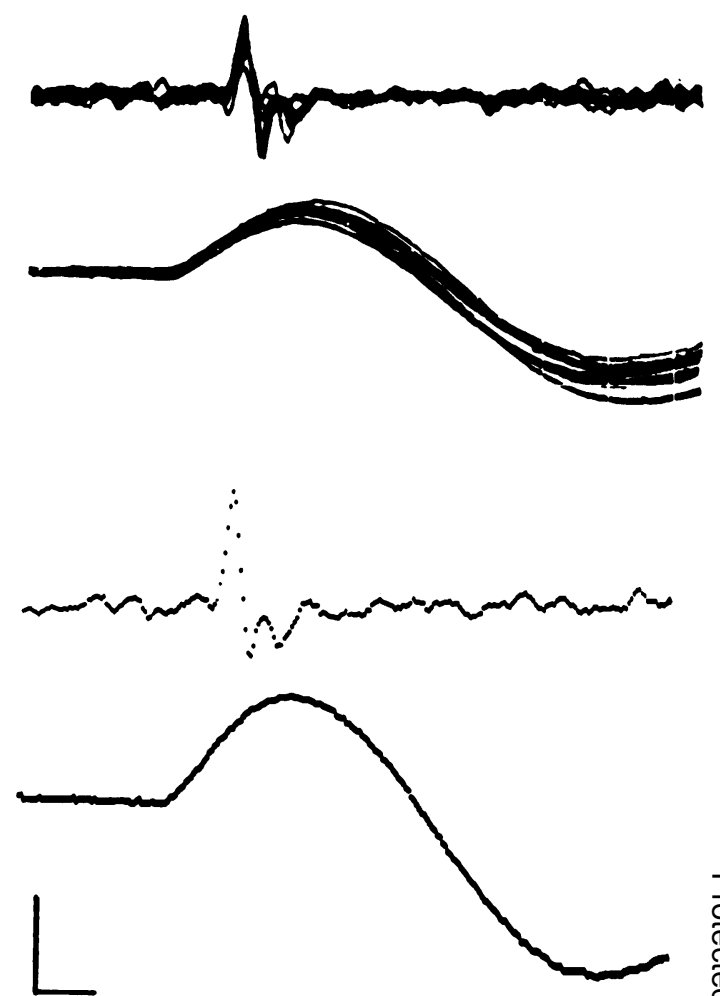

FIG. 2. The constancy of the stimulus, and the re믕 sponse of the masseter muscle. In the upper half of the figure, eight consecutive stimulus-response records have been superimposed and photographed from storage oscilloscope; the upper traces show the reflex response of the muscle, the lower traces, the displacement of the mandible (upward movement of record indicates opening ). The lower half of the figure represents the mean, in each case, of the signals shown above. These records were photographed from the signal analyser. Calibration bars represent $0.05 \mathrm{mV}$ (vertical) and $10 \mathrm{msec}$ (horizontal). Both apply to the lower figures. Peak opening displacement $0.1 \mathrm{~mm}$, added weight $3 \mathrm{~kg}$.

weight conditions are illustrated with their superimposed displacement records. Similar to its response tracing, each displacement record represents the mean of eight displacements at any one weight condition; since these records superimpose at least in their early stages, and since the delay of the masseteric reflex is of the order of $8 \mathrm{msec}$, it can be seen that at the point at which the reflex is likely to have been evoked the stimuli were virtually identical in all cases despite the variation in the load conditions. Thus the differences in amplitude of the response with increases in the voluntary activity of the 

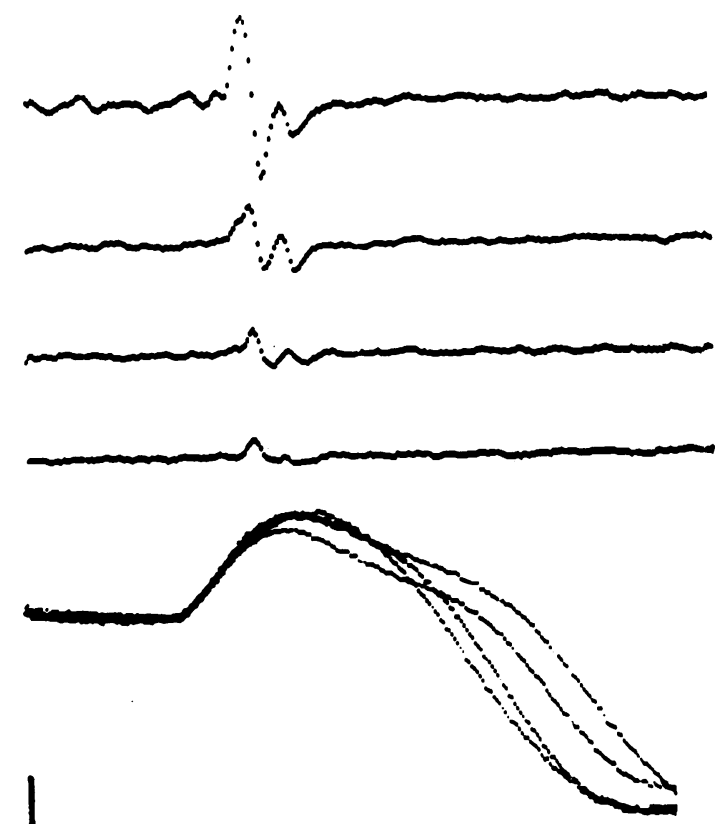

FIG. 3. Increase in the mean amplitudes of eight consecutive masseteric reflexes as a result of increasing the load supported by the mandible. The upper four traces show in each case the mean response of the muscle to increases in added weight of $0,1,2$, and $3 \mathrm{~kg}$ from below upwards. The superimposed records under the muscle responses are the mean displacement signals for the records above. Vertical calibration bar represents $0.05 \mathrm{mV}$, horizontal bar, $10 \mathrm{msec}$. Peak displacement (upward deflection represents opening) $0.1 \mathrm{~mm}$.

elevator muscles do not reflect changes in the intensity of the stimulus. When the results from all seven subjects were pooled and normalized by expressing all mean amplitudes as a proportion of the response recorded in the absence of any added weights, it was found that progressive increases in the amount of voluntary activity in the muscles caused progressive increases in the amplitude of the reflex. The data are summarized graphically in Fig. 4 where the means of the corrected, proportional changes in amplitude of the reflex have been plotted against the total force balanced by the elevator muscles as they maintained the mandible in a constant position. This force has been used as a measure of the amount of voluntary activity present in the muscles.

Clear evidence of facilitation of the masseteric reflex by the Jendrassik manoeuvre was obtained

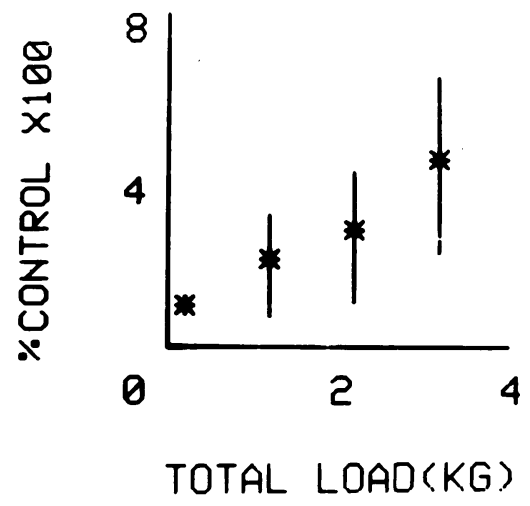

FIG. 4. Computed relationship between the proportion of the mean amplitude of the masseteric reflex to its control value (measured with zero added weight) and the total load resisted by the mandible for all seven subjects. Bars represent $\pm 1 S D$.

in only five of the seven subjects. Moreover, it was easier to demonstrate in some than in others. An example of the effect in a susceptible subject is shown in Fig. 5. Here the mean of eight consecutive responses is shown before and during the Jendrassik manoeuvre with the
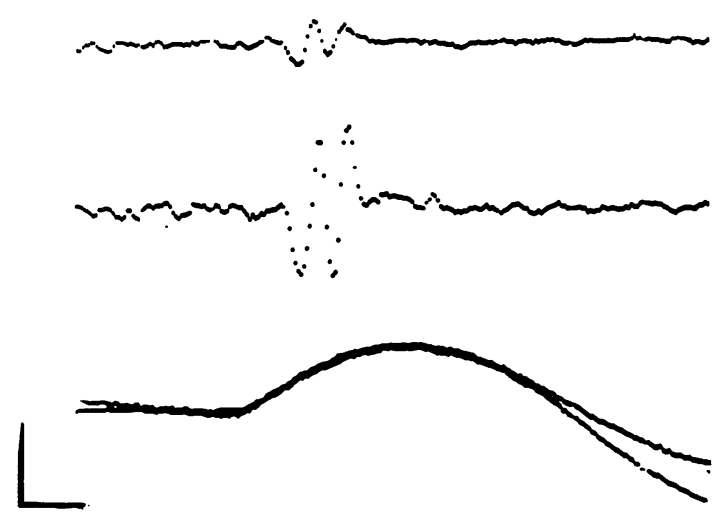

FIG. 5. Jendrassik facilitation of the masseteric reflex. The upper records show the masseteric reflex before (upper trace) and during (lower trace) strong activation of the muscles of the upper limbs (see text). The superimposed records of the displacement used to evoke the reflex in each case are shown below. Peak displacement (upward deflection of trace) $0.3 \mathrm{~mm}$. Photographed from the signal analyser.

superimposed averaged records of the stimuli used in each case. It can be seen that the displacement records are constant for both experimental runs and it therefore seems reasonable to suppose that the factor responsible for the 
facilitation of the reflex was the simultaneous contraction of muscles other than the masseter, in particular those of the upper limbs.

\section{DISCUSSION}

It has been shown in the cat that strong mechanical stimulation of the teeth may evoke a jawopening reflex (Sherrington, 1917; Hannam and Matthews, 1969) that is accompanied by reflex inhibition of the jaw-closing muscles (Sherrington, 1917; Kawamura and Fujimoto, 1958; Kidokoro, Kubota, Shuto, and Sumino, 1968). However, this reflex arc is polysynaptic (Kidokoro et al., 1968), so that even if it were activated by the taps delivered to the teeth in the present experiments any evoked effects would have appeared after the monosynaptic reflex had been completed. In addition, any long-term inhibition from a similar source due to the continued loading of the teeth by the added weights would have, if present, depressed the masseteric reflex which therefore would have shown even greater increases in amplitude with increased loads than was recorded in this study.

In the present experiments it has been assumed that the amount of voluntary contraction in the masseter muscle depends upon the weights added to the mandible, given that the latter is maintained in a constant position, and indeed this can be verified by comparing the amplitudes of the non-averaged muscle signals with the amount of added weight. However, unavoidably, there are other muscles involved in supporting the weights-for example, the temporalis and medial pterygoid muscles-and it is likely that the amount of voluntary activity in these muscles also increased with that of the masseter. It could, therefore, be argued that in the present experiments the facilitation observed as a consequence of adding weights could be explained as a Jendrassik phenomenon due to the contraction of the other elevator muscles. While not discounting a possible contribution from this source, it was clear from the experiments that in all subjects it was much easier to demonstrate facilitation of the masseteric reflex by adding weights to the mandible than it was to demonstrate Jendrassik facilitation in the same subjects when the muscles of the upper limbs were forcibly contracted. On the other hand, it is quite possible that the Jendrassik facilitation from the contraction of known synergistic, ipsilateral, and contralateral elevator muscles is greater than that from more distant muscle masses. Therefore the facilitation of the masseteric reflex observed in the loading experiments may have been due to Jendrassik facilitation by the contraction of synergistic muscles, autogenetic facilitation caused by the voluntary contraction of the muscle itself, or to a combination of both. In their experiments on the ankle jerk reflex, Ott and Gassel (1969) reported progressive increases in amplitude of the reflex when the voluntary activity in the contralateral triceps surae muscle was increased, but when the ipsilateral muscle was used an increase occurred only with mild degrees of voluntary contraction; further increases in contraction progressively inhibited the reflex. They suggested that the inhibition could be related to a number of factors including occlusion of the reflex due to active and refractory motor units, autogenic inhibition from Golgi tendon organs, and recurrent collateral inhibition. Thus on the basis of these observations it would seem that the most likely cause of masseteric reflex facilitation is the contraction of synergistic muscles, particularly those on the contralateral side, with sufficient intensity tod

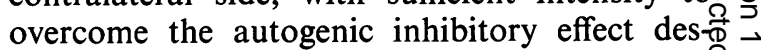
cribed by Ott and Gassel. However, in their experiments, an automatic solenoid hammer secured at a fixed distance above the Achilles tendon, was used to deliver a constant blow to the tendon. No attempt was made to monitor the displacement of the heel as a result of this blow before the contraction of the triceps surae muscle. It is therefore probable that with an increased amount of voluntary contraction of the muscle there would be less lengthening of the muscle for a given blow applied to the tendon. Since muscle spindles respond to changes in length of the muscle rather than to changes in tension, there would be a smaller effective stimulus to the spindles during increased background contraction (given that the blow was constant) and the ankle jerk reflex would be reduced in amplitude. For this reason, it is still not clear whether, at high levels of background activity, reflex contraction of the same muscle is inhibited or facilitated. Thus, although in the present experiments the displacement of the mandible was kept constant and only progressive facilitation was observed, it is yet a problem to decide whether this enhancement of the masseteric reflex was due to autogenetic factors, synergistic factors, or both.

Insofar as the results of this work relate to the 
activity in the masseter muscle after tooth contact during mastication, it would seem that at least two processes are responsible for the amplitude of the masseteric reflex evoked in these circumstances. The more vigorously the jaws are brought together, the greater will be the impact of the teeth, the greater the stimulus to the muscle spindles, and the greater the amplitude of the reflex. In addition, the more vigorously the jaws are closed the more activity will be present in the masseter and other elevator muscles at the time the reflex is evoked and therefore, as the present experiments have shown, the more would be the facilitation of the response due to this effect alone.

The author wishes to thank Mr. Robin Forrest for his assistance during these experiments. The work was supported by the Medical Research Council of Canada.

\section{REFERENCES}

Ahlgren, J. (1969). The silent period in the EMG of the jaw muscles during mastication and its relationship to tooth contact. Acta Odontologica Scandinavica, 27, 219-227.

Beaudreau, D. E., Daugherty, W. F. Jr., and Masland, W. S. (1969). Two types of motor pause in masticatory muscles. American Journal of Physiology, 216, 16-21.

Brenman, H. S., Black, M. A., and Coslet, J. G. (1968). Interrelationship between the electromyographic silent period and dental occlusion. Journal of Dental Research, 47, 502 .
Griffin, C. J., and Munro, R. R. (1969). Electromyography of the jaw-closing muscles in the open-close-clench cycle in man. Archives of Oral Biology, 14, 141-149.

Hagbarth, K. E. (1962). Post-tetanic potentiation of myotatic reflexes in man. Journal of Neurology, Neurosurgery, and Psychiatry, 25, 1-10.

Hannam, A. G., Matthews, B., and Yemm, R. (1969). Changes in the activity of the masseter muscle following tooth contact in man. Archives of Oral Biology, 14, 14011406.

Hannam, A. G., Matthews, B., and Yemm, R. (1970). Receptors involved in the response of the masseter muscle to tooth contact in man. Archives of Oral Biology, 15, 1724.

Hannam, A. G., and Matthews, B. (1969). Reflex jaw opening in response to stimulation of periodontal mechanoreceptors in the cat. Archives of Oral Biology, 14, 415-419

Hoffmann, P. 1922. Untersuchungen über die Eigenreflexe (Sehnenreflexe) menschlicher Muskeln. Springer: Berlin.

Kawamura, Y., and Fujimoto, J. (1958). A study on the jaw opening reflex. Medical Journal of Osaka University, 9 , 377-387.

Kidokoro, Y., Kubota, K., Shuto, S., and Sumino, R. (1968). Reflex organization of cat masticatory muscles. Journal of Neurophysiology, 31, 695-708.

Matthews, B., and Yemm, R. (1970). A silent period in the masseter electromyogram following tooth contact in subjects wearing full dentures. Archives of Oral Biology, 15, 531-535.

Ott, K. H., and Gassel, M. M. (1969). Methods of tendon jerk reinforcement. Journal of Neurology, Neurosurgery, and Psychiatry, 32, 541-547.

Schaerer, P., Stallard, R. E., and Zander, H. A. (1967). Occlusal interferences and mastication: an electromyographic study. Journal of Prosthetic Dentistry, 17, 438-449.

Sherrington, C. S. (1917). Reflexes elicitable in the cat from pinna, vibrissae, and jaws. Journal of Physiology, 51, 404431. 